\title{
The Use of Radiofrequency Energy in Pediatric Cardiology
}

\author{
PARVIN C. DOROSTKAR, M.D. and MACDONALD DICK, II, M.D.* \\ From the Division of Pediatric Cardiology, University of California, San Francisco, San Francisco, California; and the *Department of \\ Pediatrics, University of Michigan, Ann Arbor, Michigan
}

\begin{abstract}
The use of radiofrequency energy for the treatment of supraventricular tachycardia in pediatric patients has gained widespread acceptance, especially for tachyarrhythmias associated with palpitations, dizziness, presyncope or syncope, cardiomyopathy, and cardiac arrest. Ablation of the substrate supporting atrioventricular reentry, atrioventricular node reentry, and automatic atrial tachycardia yields a $90 \%-98 \%$ success rate with low incidence $(<1 \%)$ of complications and adverse side-effects. Ablation of intra-atrial reentry, including atrial flutter and fibrillation. appears to be promising and would be a significant advance in the management of patients following extensive atrial surgery for congenital heart disease. Radiofrequency energy is also used to treat various forms of idiopathic ventricular tachycardia. Finally, radiofrequency energy has been extended to control the ventricular rate associated with malignant atrial tachycardia by either modification or ablation of the atrioventricular node. and subsequent pacemaker implant. Long-term outcome of radiofrequency ablation is unknown, but the shortto-intermediate (1-5 yrs) outcome is excellent, with low recurrence rate of the tachycardia, no proarrhythmic effect, and excellent clinical state. (J Interven Cardiol 1995;8:557-568)
\end{abstract}

\section{Introduction}

During the past half decade, transcatheter radiofrequency energy delivered to conducting pathways or ectopic foci within the heart has emerged as the primary and often completely corrective treatment for many forms of tachyarrhythmias in the young. The energy, delivered in the form of alternating $(500 \mathrm{~Hz})$ current between a 4-mm catheter tip electrode and a large electrode patch on the patient's back, produces resistive heating of the catheter tip electrode, resulting in local tissue coagulation necrosis and cessation or alteration of conduction within and immediately adjacent to the myocardial lesion. The size of the lesion generated depends on the ablating electrode size, the tissue temperature reached $\left(60^{\circ} \mathrm{C}-80^{\circ} \mathrm{C}\right)$, the duration of energy application, degree of tissue contact, and the stability of the ablating electrode tip during energy delivery. ${ }^{1}$ Most commonly, radiofrequency energy is directed at an accessory pathway or a slow atrioventricular nodal pathway supporting supraventricular

Address for reprints: Parvin Dorostkar M.D., Room C346, Box 0632. University of California, San Francisco, San Francisco, CA 94143-0632. Fax: (415) 476-3112. tachycardia. ${ }^{1-9}$ It has also been directed at ectopic foci of both atrial ${ }^{2.10}$ and ventricular tachycardia ${ }^{11,12}$ as well as intra-atrial arrhythmias such as atrial flutter, especially following extensive atrial surgery for complex congenital heart disease. ${ }^{13,14}$ In addition, intractable atrial or junctional tachycardias have been successfully treated with radiofrequency ablation or modification of the atrioventricular node ${ }^{15-17}$ followed by ventricular pacemaker implant.

\section{Incidence and Mechanisms of Supraventricular Tachycardia}

Supraventricular tachycardia is the most common sustained cardiac arrhythmia in children. It occurs in approximately 1 out of $250-1,000$ children. Reentrant supraventricular tachycardia accounts for approximately $90 \%$ of supraventricular tachycardia in children; automatic, i.e., ectopic, compromise the remaining $10 \% .{ }^{18}$ The most common type of supraventricular tachycardia in children is atrioventricular reentry tachycardia involving the atrium, atrioventricular node, His-Purkinje system, ventricular myocardium, 
Table 1. Tachycardias Treated with Radiofrequency Energy

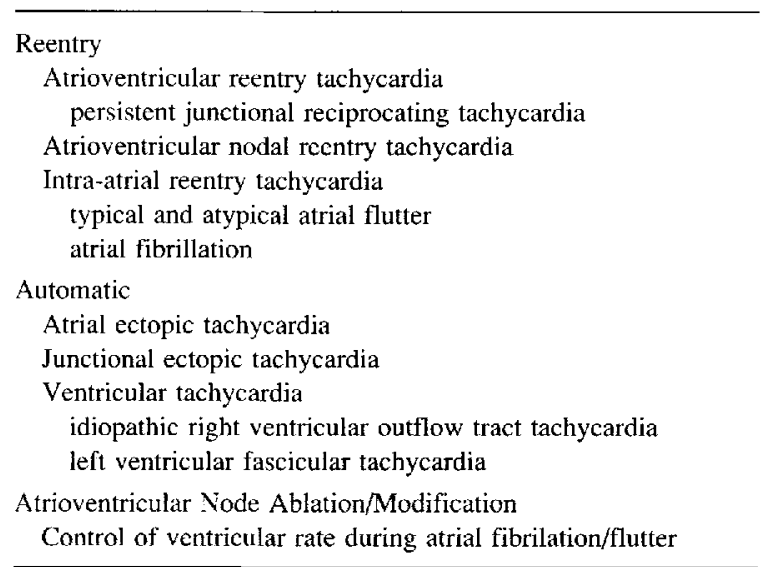

and accessory pathway; comprising approximately $50 \%-60 \%$ of reentrant supraventricular tachycardia in children and young adults (Table 1).

Atrioventricular reentry tachycardia is supported by one or more manifest or concealed accessory connections. In patients with manifest accessory connections, the impulse may conduct antegrade through both the normal atrioventricular node and the accessory connection, producing the typical short $\mathrm{P}-\mathrm{R}$ interval and delta wave during normal sinus rhythm. Most commonly, during tachycardia, these patients conduct antegrade through the nommal atrioventricular node and retrograde through the accessory connection, therefore inscribing a narrow QRS tachycardia. In contrast, in patients with concealed accessory connections, conduction occurs only retrograde through the accessory connection. Thus, during tachycardia, the antegrade conduction occurs through the atrioventricular node, whereas retrograde conduction occurs through the accessory connection. During tachycardia these two types of supraventricular tachycardia are indistinguishable. Occasionally, during supraventricular tachycardia, rate related bundle branch aberrancy occurs, therefore inscribing a wide QRS complex tachycardia. In a less frequent event ( $3 \%$ in our experience), antegrade conduction occurs through the manifest accessory connection with retrograde conduction through the atrioventricular node, known as antidromic reciprocating tachycardia (also inscribing a wide QRS complex). The remaining forms of reentrant supraventricular tachycardia are supported by dual atrioventricular node pathways, sinoatrial node reen- try, or intra-atrial reentry including atrial flutter and atrial fibrillation.

\section{Indications for Radiofrequency Ablation}

When supraventricular tachycardia is frequent, sustained or incessant, and associated with significant symptoms, such as palpitations, faintness, dizziness, presyncope, syncope, and rarely cardiac arrest, or associated with diminished left ventricular function suggestive of cardiomyopathy, intervention is required. Pharmacological control of these tachycardias is difficult, potentially life-long, and associated with side-effects. Thus, because of its safety and efficacy, radiofrequency energy has largely replaced pharmacological treatment of many forms of supraventricular tachycardia in the young. It is important to note that, as outlined by the Pediatric Electrophysiology Society report, ${ }^{3}$ failure rates and serious complications are inversely related to patient size; thus, radiofrequency energy should be reserved for those children $>15 \mathrm{~kg}$ in weight, and used in smaller infants only for highly individualized indications by the most experienced operators.

\section{Method of Radiofrequency Ablation}

After appropriate informed consent, patients are brought to the electrophysiology laboratory in the postabsorptive state and receive either conscious sedation or general anesthesia. Most patients may be studied with 5Fr, 6Fr, or 7Fr electrode catheters; for infants and smaller children electrode catheters as small as 3Fr are available for recording and mapping. Standard programmed stimulation is performed to evaluate atrioventricular conduction and determine the mechanism of tachycardia. The continuously monitored bipolar ECGs are stored on either a strip chart recorder moving at paper speeds of $50-100 \mathrm{~mm} / \mathrm{sec}$ or on computer driven systems with optical disc storage. Electrode catheters are placed in the high right atrium, coronary sinus, His-bundle area, and right ventricular apex for recording of the bipolar clectrograms and delivery of extrastimuli. If coronary sinus cannulation is not possible (and there is no patent foramen ovale), an electrode catheter can be advanced into the left pul- 


\section{RADIOFREQUENCY FNERGY IN PEDIATRIC CARDIOLOGY}

monary artery to distinguish between left- and rightsided retrograde atrial activation, and thus between left- and right-sided accessory pathways.

If the pathway is left sided, transseptal entry into the left atrium may be used. After placement of the catheters, including the transseptal catheter, the patient is anticoagulated with $1,000 \mathrm{U} / \mathrm{kg}$ of heparin (maxi mum up to $3,000 \mathrm{U} /$ dose) which is administered every 90-120 minutes pending an activated clotting time. Radiofrequency current is delivered at 20-40 watts (W) for 10-60 seconds at a frequency of $500 \mathrm{kHz}$. The most useful electrogram patterns indicating a potentially good site for ablation include short antegrade and retrograde conduction times between the atrial electrogram and ventricular depolarization, local ventricular preexcitation (the earliest ventricular excitation recorded through the distal pair of electrodes on the ablating catheter and usually preceding ventricular excitation recorded through the surface ECG), a suspect Kent potential in patients with manilest pathways, but not concealed pathways, and prompt response to radiofrequency energy application. ${ }^{19,20}$ A small decrease in impedance during radiofrequency energy application confirms good current flow and tissue contact. ${ }^{21}$ The use of a thermistor catheter may help to assess myocardial tissue contact and confirm temperatures sufficient to abolish anomalous conduction or ectopic foci. If tachycardia or preexcitation is not affected within 10 seconds, delivery of radiofrequency energy is terminated and a new site identified. Thirty to 60 minutes after apparent successful ablation, atrial and ventricular pacing and extrastimulation are repeated to ensure successful ablation as well as persistence of normal antegrade and retrograde conduction. Follow-up in 4-6 weeks and 1 year later to evaluate recurrence of an accessory connection as well as to document absence of any other arrhythmias is advised.

Atrioventricular Reentry Tachycardia. In patients with manifest accessory connections, the surface ECG can be used as a general guide for the localization of the accessory pathway. ${ }^{22,23}$ Once the mechanism of tachycardia has been confirmed to be dependent upon an accessory pathway, a mapping catheter can be moved in a systematic fashion around the atrioventricular ring for more accurate determination of pathway localization. In general, a large ventricular signal accompanied by a smaller atrial electrogram confirms proximity to the atrioventricular annulus, but not necessarily to the accessory pathway. The site of maximal local ventricular preexcitation, the earliest site of retro- grade atrial activation during tachycardia or ventricular pacing, and a possible Kent fiber potential define three criteria for locating proximity to the accessory pathway and thus a potentially successful site for ablation. ${ }^{19,20}$ Because posteroseptal pathways lie near the normal atrioventricular conduction pathways, their antegrade and retrograde activation patterns are difiicult to distinguish from patterns resulting from normal pathways. Therefore, in patients with posteroseptal pathways, cannulation of the coronary sinus and careful mapping around the mouth of the coronary sinus and the tricuspid valve annulus adjacent to the atrioventricular node is essential.

For right-sided pathways, the challenge is to achieve good myocardial tissue contact. For this purpose, several authors have proposed using a long multipurpose sheath or long curved sheaths for increased catheter stabilization. ${ }^{24}$ The deflectable ablation catheter tip can then be advanced through the sheath and more easily stabilized on the right free-wall atrioventricular groove where the earliest activation may be recorded. Right-sided pathways are approached through either the inferior or superior vena cava from above the tricuspid valve in the right atrium or across and under the tricuspid valve in the right ventricle. Since anterior septal pathways lic close to the His bundlc, although separated by the central fibrous body, mapping of suspected pathways in this region may be performed using a catheter advanced from the superior vena cava, internal jugular vein, or inferior vena cava. Adjusting the gain of the mapping electrograms so that they equal the gain of the recording electrograms facilitates identification of a possible His electrogram at the potential ablating site and thus allows for safe radiofrequency energy application.

For successful ablation of left-sided pathways, two approaches have been used. The first method is from above the atrioventricular ring, usually reached from the left atrium by the transseptal approach. A long exchange wire is advanced into the superior vena cava and a flushed transseptal sheath, and dilator are advanced over it. The exchange wire is then removed and the transseptal thin trochar is advanced into the sheath and dilator, but not beyond the tip of the dilator. A 5-ce syringe filled with contrast is attached to the trochar's hub. The trochar, sheath and dilator are then withdrawn under fluoroscopic and simultaneous transthoracic or transesophageal echocardiographic guidance, at a unit, so that the curved end of the trochar is directed posteriorly (indicated by a posterior orienta- 


\section{DOROSTKAR AND DICK}

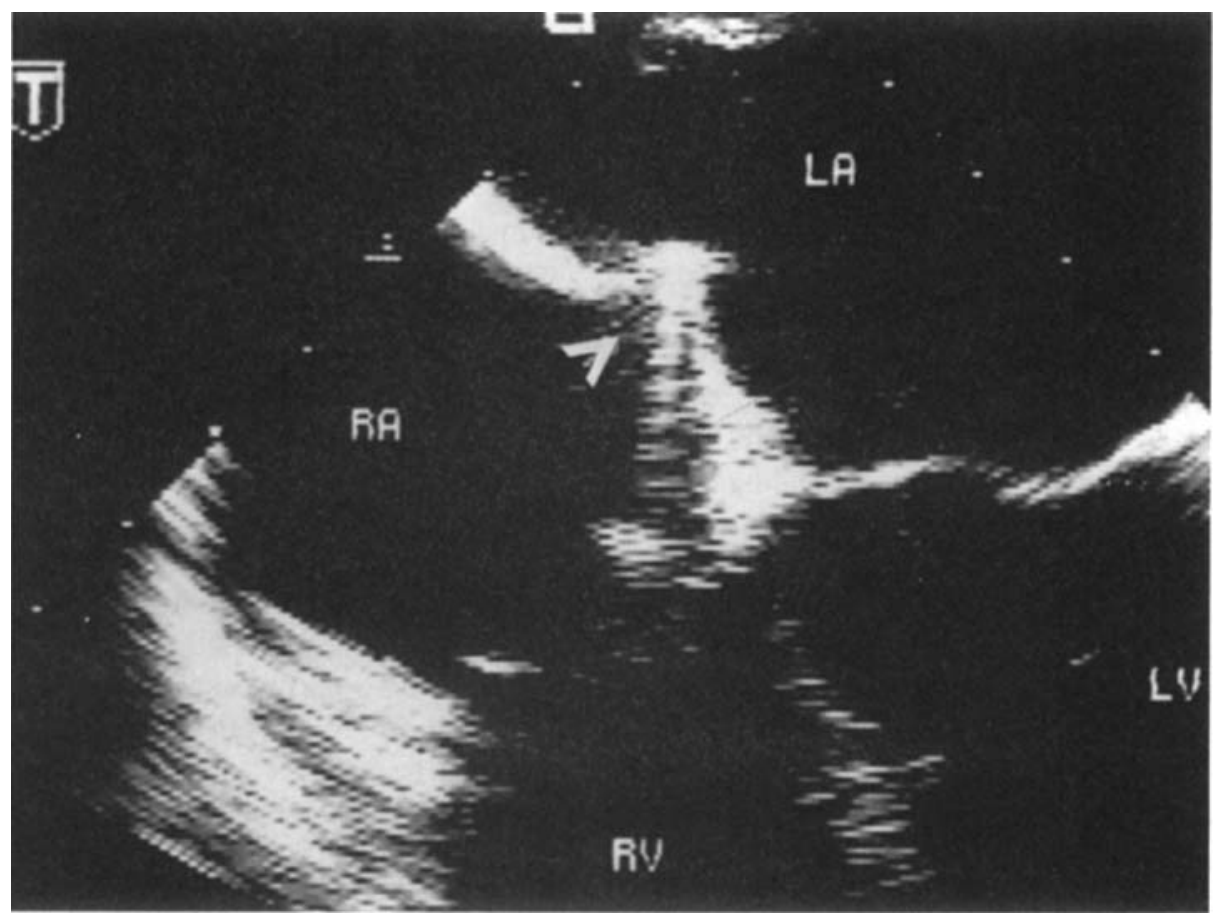

Figure 1. Transesophageal echocardiographic view showing tenting of the atrial septum by the transseptal trochar (arrow) just prior to passage across the septum. $L A=$ left atrium; $L V=$ left ventricle; $R A=$ right atrium; $R V=$ right ventricle.

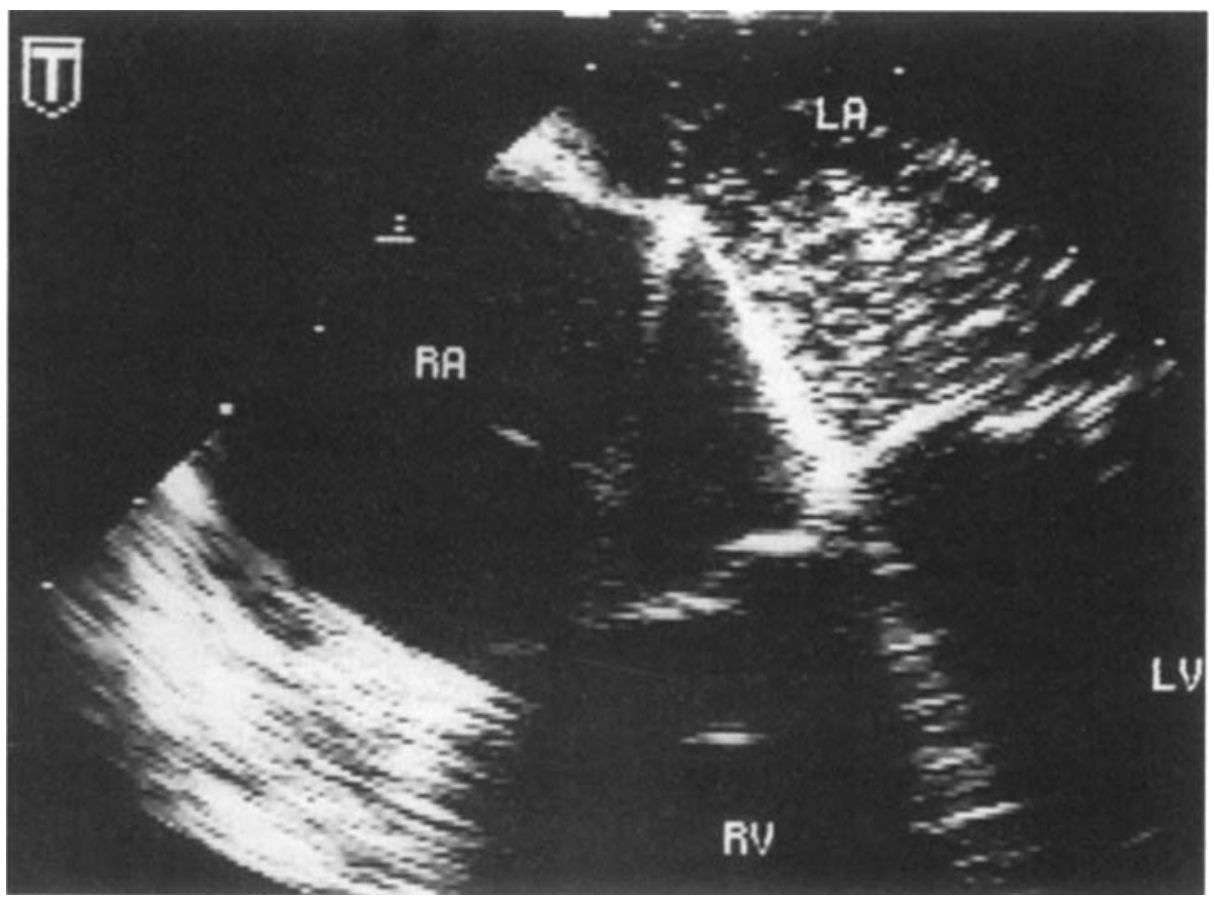

Figure 2. Transesophageal echocardiographic view after contrast administration (arrow) confirming that the transseptal trochar has passed into the left atrium. $\mathrm{LA}=$ left atrium; $\mathrm{LV}=$ left ventricle; $\mathrm{RA}=$ right atrium; $\mathrm{RV}=$ right ventricle. 
tion of the indicator located at the trochar's hub) until the tip of the unit moves posteriorly over the aortic knob and into the fossa ovalis (Fig. 1). The tip of the transseptal trochar is then advanced a few millimeters out of the unit into the atrial septum, indicated by the sensation of resistance and confirmed by the injection of a small amount of contrast producing a radioopaque stain in the atrial septum. The transseptal trochar, dilator, and sheath, as a unit, is then advanced through the atrial septum into the left atrium (Fig. 2). Once in the left atrium, the transseptal sheath and dilator are advanced over the trochar so as to be completely in the left atrium; the dilator and needle are then slowly (to avoid cavitation) removed and the mapping/ ablation catheter is slowly advanced through the sheath to the left atrium. The tip of the mapping/ablation catheter is gently maneuvered in small increments around the mitral valve annulus and the aforementioned criteria for pathway localization are used to locate a potential ablation site (Figs. 3-6). Only rarely is it necessary to advance the ablation catheter into the left ventricle and under the mitral valve annulus, where successful ablation can be achieved. Invasive monitor- ing of arterial pressure and/or localization of the aortic root by placing a pigtail catheter in the ascending aorta is usually not required and should be individualized for each patient. The second method is the retrograde approach, wherein the ablation catheter is advanced retrograde through the femoral artery, around the aortic arch, and across the aortic valve into the left ventricle. Mapping of a lleft-sided accessory connection is then performed by advancing the ablation catheter under the mitral valve annulus. Occasionally, using this approach, the catheter tip can be manipulated through the mitral valve and made to rest on the mitral valve annulus on top of the mitral valve. ${ }^{24}$ Although there is no reported difference in success rates between the two methods, an increased incidence of Doppler detected valvar regurgitation has been noted in association with the retrograde approach ${ }^{25}$ and has been essentially eliminated with the transseptal approach. ${ }^{26,27}$

A unique type of atrioventricular reentrant tachycardia is persistent junctional reciprocating tachycardia. This tachycardia is characterized by a long R-P and short P-R interval, negative $\mathrm{P}$ waves in leads II, III, and AVF, and is often incessant. It is supported by a

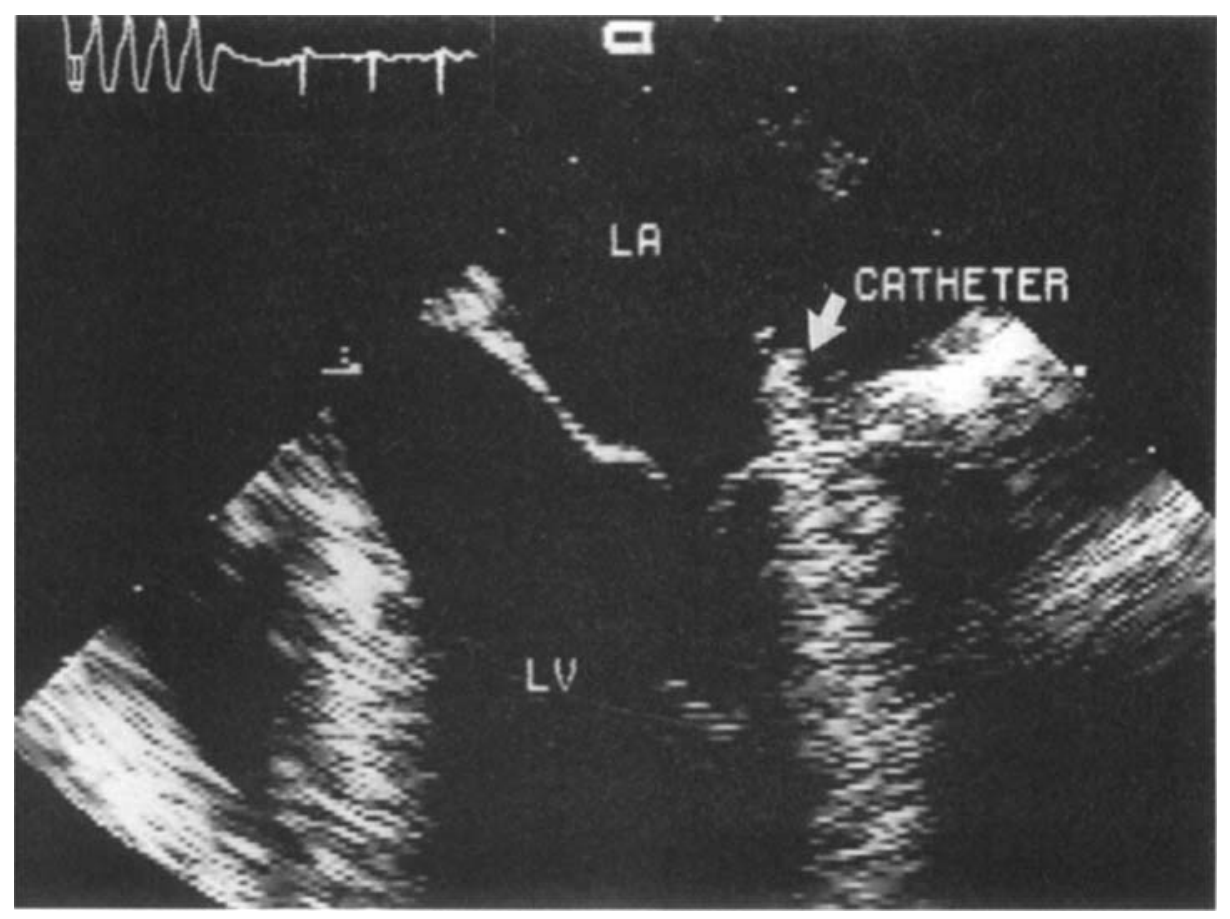

Figure 3. Transesophageal echocardiographic vicw of the transseptal catheter (arrow) at the left lateral atrioventricular ring during delivery of radiofrequency energy. Note cessation of wide QRS tachycardia followed by normal sinus rhythm on the EC( (left upper inset). LA - left atrium; LV = left ventricle. 


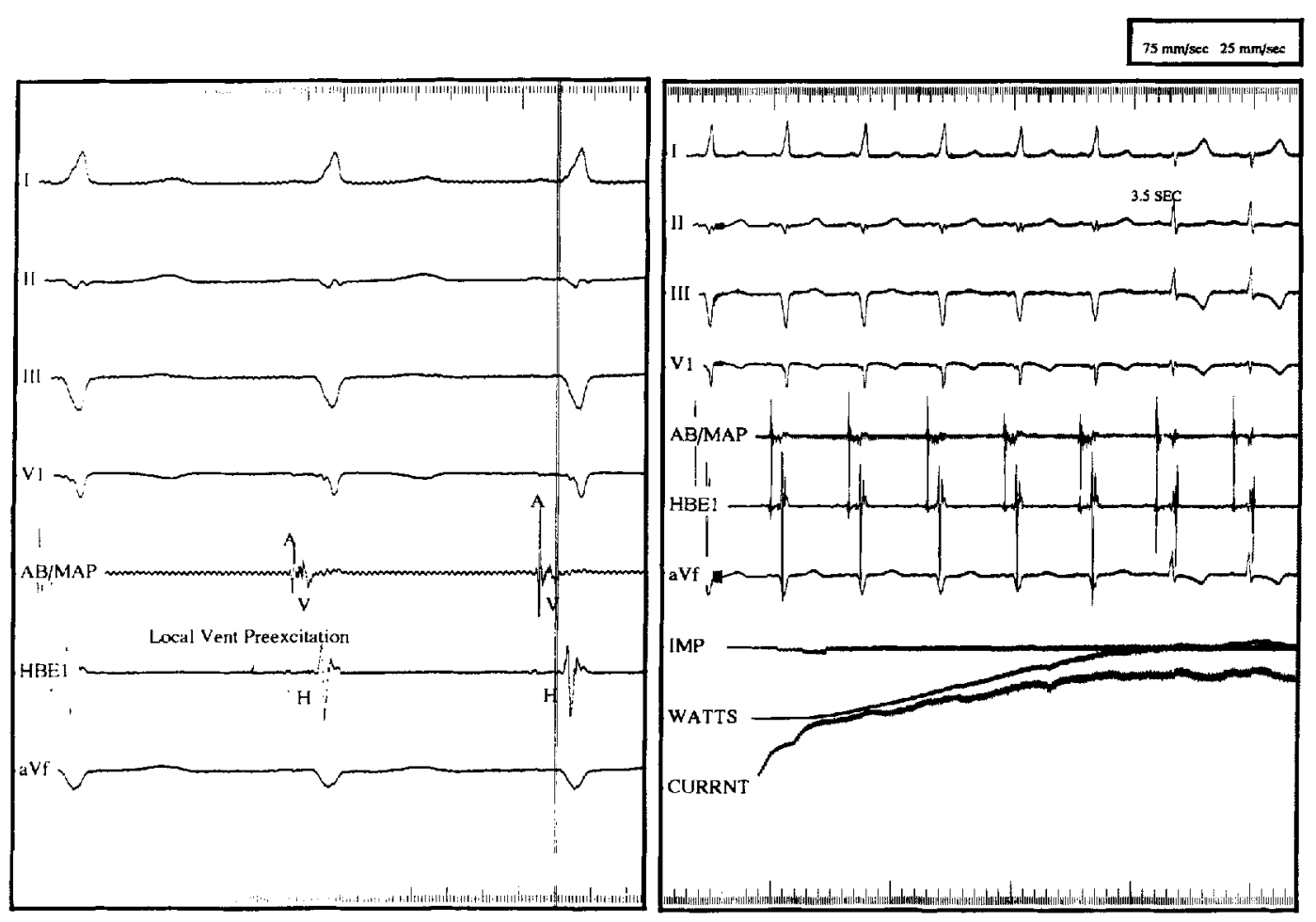

Figure 4, Left hand panel: Local ventricular preexcitation is noted by the $\mathrm{V}$ wave recorded through the $\mathrm{AB} / \mathrm{MAP}$ catheter tip electrodes prior to the onset of the QRS complex (shown by vertical line). Right hand panel: Loss of preexcitation 3.5 seconds after onset of radiofrequency current flow. $\mathrm{A}=$ atrial electrogram; $\mathrm{AB} / \mathrm{MAP}=$ ablation/map electrogram recorded through catheter tip electrodes: aVF = lead aVF; CURRNT = current $(\mathrm{mA})$ of radiofrequency energy delivered; $\mathrm{H}=$ His-bundle electrogram; HBE1 = His-bundle electrogram; IMP = impedence (ohms) to radiofrequency energy delivered; $\mathrm{V}=$ ventricular electrogram; Vent $=$ ventricular; $\mathrm{VI}=$ lead V1; WATTS $=$ watts of radiofrequency energy delivered; I, II, III = leads I, II, III.
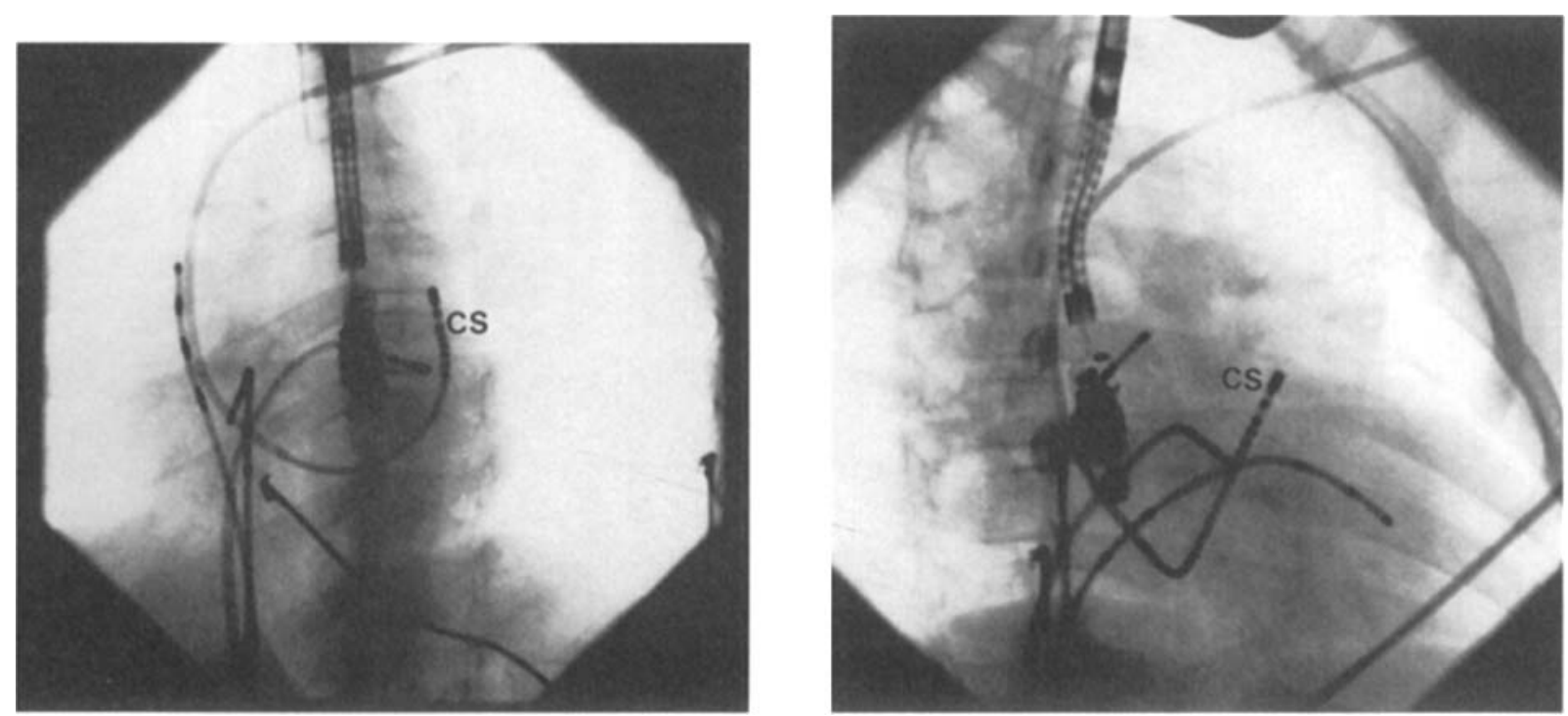

Figure 5 and Figure 6. Left anterior oblique and right anterior oblique projections demonstrating transesophageal echocardiographic probe, coronary sinus catheter, His-bundle catheter, and the ablating catheter within the left atrium on the atrioventricular ring on the left lateral site of the accessory pathway. 


\section{RADIOFREQUENCY ENERGY IN PEDIATRIC CARDIOLOGY}

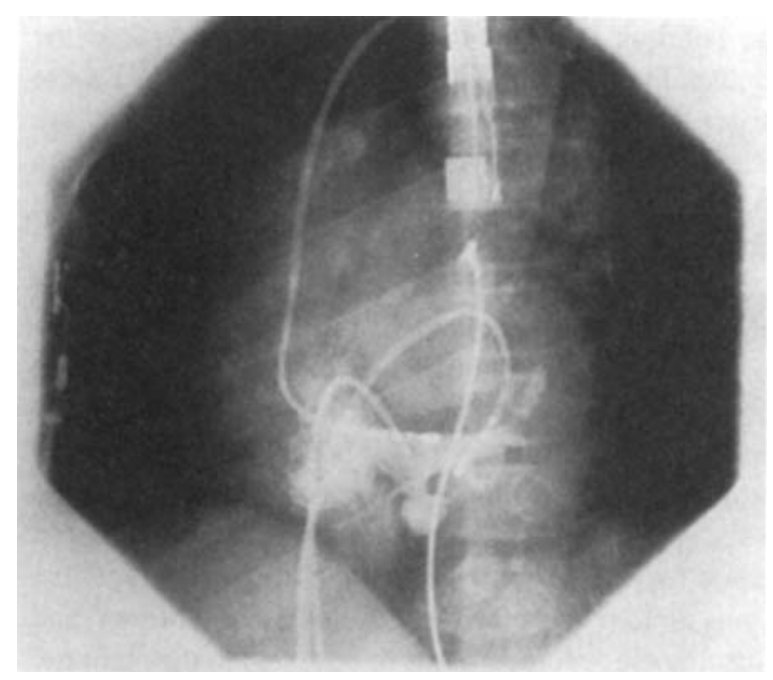

Figure 7. Coronary sinus venogram demonstrating an anomalous coronary sinus diverticuli-like structure in which an accessory pathway was located and successfully ablated.

concealed, slowly, retrogradely conducting pathway with decremental conduction properties that usually returns to the atrium around the mouth of the coronary sinus. This pathway can be ablated using similar mapping and ablation techniques to those described above. ${ }^{28-30}$

In our experience, recurrence rate of the pathway and tachycardia is $1.5 \%$ with left-sided pathways, but $20 \%$ with right-sided pathways. Repeated sessions are warranted since they result in $90 \%-95 \%$ success. If ablation of an accessory connection is difficult, especially on the left side, the coronary sinus should be examincd by coronary sinus venogram for possible coronary sinus anomalies, such as thin walled extracoronary veins or diverticuli (Fig. 7), since accessory pathways may pass through them. When the mapping electrode is placed in these anomalies, the close proximity of the electrode to the pathway produces a characteristic large Kent fiber electrogram identifying a potential successful site. In these patients, delivery of low (gradual increase to $\leq 15 \mathrm{~W}$ ) radiofrequency energy within the coronary sinus at the mapped site is usually sufficient to interrupt anomalous conduction. Finally, a single catheter retrograde approach to manifest left sided accessory connections has been described. ${ }^{31}$

Atrioventricular Nodal Reentry Tachycardia. In our experience, patients with atrioventricular nodal reentry tachycardia comprise approximately $15 \%$ of young patients undergoing radiofrequency ablation for supraventricular tachycardia. In the majority of these patients, the slow pathway provides the antegrade limb of the circuit, whereas the fast pathway is the retrograde limb (i.e., the slow-fast or common type of atrioventricular node reentry tachycardia). Less commonly, the fast pathway may provide the antegrade limb whercas the slow pathway serves as the retrograde limb. Atrioventricular node reentry is treated by directing radiofrequency energy through the electrode tip of the ablation catheter to a limb of reentrant circuit found within the bounds of the triangle of Koch. Early efforts were directed toward modifying the fast pathway, lying anteriorly and superiorly to the atrioventricular node at the apex of the triangle of Koch. ${ }^{89}$ However, because of the risk of complete heart block when delivering energy to the fast pathway, attention shifted to the slow pathway, located posterior to the atrioventricular node near the coronary sinus. ${ }^{89}$ Successful atrioventricular nodal modification is associated with fractionated atrial electrograms and larger ventricular electrograms (compared to atrial electrograms: 1:2 A:V ratio) as well as the appearance of junctional tachycardia during energy application. Because of the aforementioned risk of complete heart block, ${ }^{8}$ atrioventricular node modification should be advised and undertaken very cautiously in the pediatric population. To avoid this potential risk, a repeat procedure at a later date may be a more reasonable option in small children, rather than continuing application of energy to the small area within the triangle of Koch adjacent to the normal atrioventricular node and penetrating His bundle. Overall, modification of the slow pathway has produced excellent $(90 \%-100 \%$ of patients remain tachycardia-free) results. After the procedure, programmed atrial and ventricular stimulation with isoproterenol should be performed to document absence of inducible tachycardia and persistence of normal atrioventricular node function.

Ectopic Atrial Tachycardia. Ectopic or automatic atrial tachycardia may be either incessant or intermittent, ${ }^{10}$ may result in tachycardia related cardiomyopathy, and is often difficult to control with medical therapy. ${ }^{32}$ Local atrial activation times recorded during the tachycardia from the distal electrode pair of the mapping/ablation catheter are compared to the onset of the surface $P$ wave to generate an atrial activation map. Fine adjustments of the mapping catheter will then yield the earliest atrial activation site, usually pre- 


\section{DOROSTKAR AND DICK}

ceding the onset of the surface $\mathrm{P}$ wave by $>15-20$ msec. Radiofrequency energy is then applied at that site at a lower power $(20-25 \mathrm{~W})$ for $10-30$ seconds (Fig. 8). Most automatic foci driving the tachycardia accelerate for 5-10 seconds upon application of thermal energy (a few will slow and some terminate abruptly) and then are extinguished. The patients should be observed after successful ablation since ectopic foci exhibit a high recurrence rate.

Intra-Atrial Reentry Tachycardia. Typical (sawtoothed pattern in leads II, III, and AVF, and flutter cycle length of $200 \mathrm{msec}$ ) and atypical atrial flutter, as well as reentrant atrial tachycardia have been successfully treated with radiofrequency energy application. ${ }^{13,14}$ In these patients, concealed entrainment is used to identify the area of slow conduction of reentrant circuits located near anatomic barriers such as the isthmus between the coronary sinus os and the tricuspid valve, surgical scars, or suture lines. At these target sites, 20-40 W of radiofrequency energy are delivered during tachycardia for 20-60 seconds (Fig. 9). Procedure end point is the inability to reinduce atrial reentry tachycardia.

Sinus Node Reentry Tachycardia. Treatment of sinoatrial node reentrant tachycardia has been successfully achieved by radiofrequency energy application around the area of the crista terminalis adjacent to the sinoatrial node in older patients. ${ }^{33}$ Intracardiac echocardiographic imaging has been performed in order to assist appropriate sites of radiofrequency energy application for patients with sinus node reentry tachycardia. Sinus node reentry tachycardia is rare in children, and thus this procedure remains to be tested in the pediatric population.

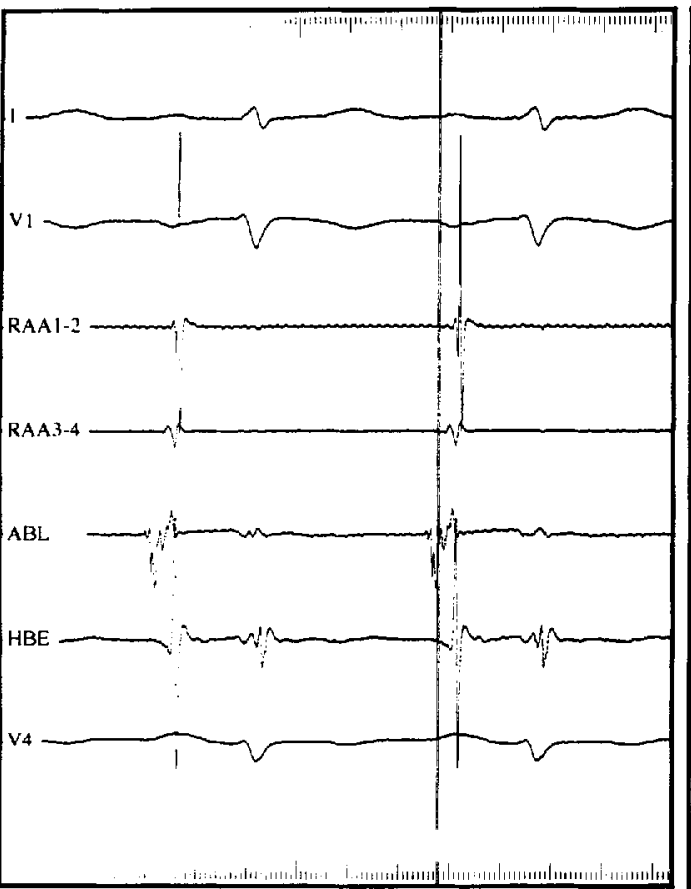

$100 \mathrm{~mm} / \mathrm{sec} 25 \mathrm{~mm} / \mathrm{sec}$

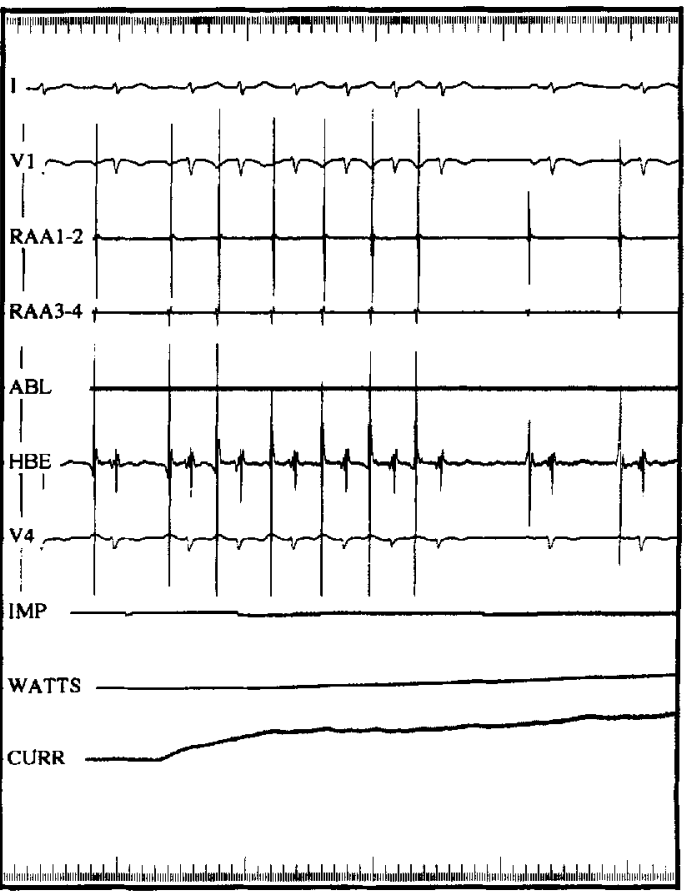

Figure 8. Left hand panel: Atrial automatic (ectopic) tachycardia at a cycle length of $510 \mathrm{msec}$. Note early atrial activation on the ABL electrogram (indicated by atrial activity before the vertical line) compared to other sites and compared to the $P$ waves. The ABL electrode catheter is located on the mid-posterior wall of the left atrium confirming an ectopic focus driving this rhythm. Right hand panel: The atrial ectopic thythm is initially accelerated and then extinguished by radiofrequency current, followed by resumption of sinus rhythm. $\mathrm{ABL}=$ distal atrial electrogram; CURR $=$ current $(\mathrm{mA})$; HBE $=$ His-bundle electrogram; IMP $=$ impedence $($ ohms) to radiofrequency energy delivered; RAA1-2,3-4 = right atrial appendage electrogram $1-2$ and $3-4$; V1, V4 Lead $=$ V1, V4; WATTS = watts of radiofrequency energy delivered; 1 = lead 1. 


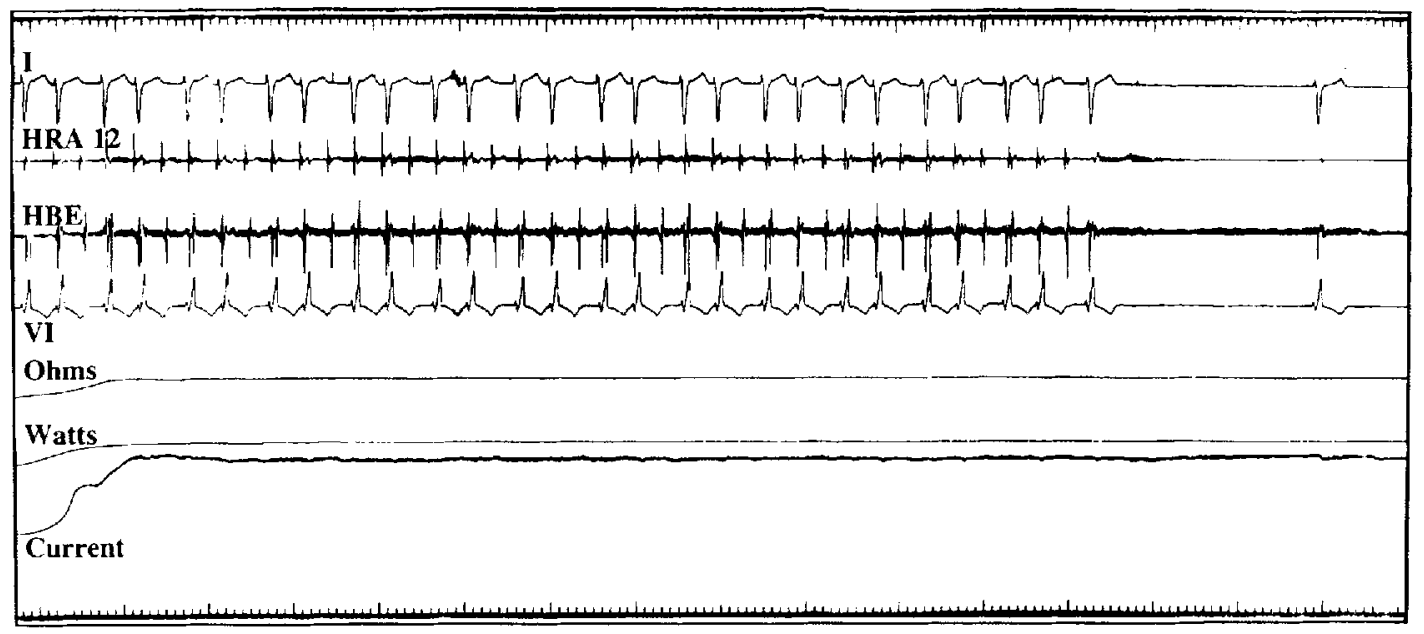

Figure 9. Ablation of atrial flutter.

Ablation of the Atrioventricular Node. Atrioventricular node ablation can be used to treat patients with intractable malignant atrial tachyarrhythmias such as atrial flutter or atrial fibrillation with fast atrioventricular nodal conduction. In patients with congenital heart disease, transvenous access to the atrioventricular node and His bundle may be difficult since the atrioventricular node and His bundle may be excluded from the systemic venous atrium by operative design (such as transposition of the great arteries after Mustard operation or single ventricular physiology after Fontan operation). In these patients, atrioventricular node $a b-$ lation has been successfully achieved using the retrograde aortic route to the atrioventricular node/His bundle. ${ }^{15,16}$

\section{Ventricular Tachycardia.}

Right ventricular tachycardia. Successful ablation of right ventricular outflow tachycardia, characterized by left bundle branch morphology and an inferior axis and associated with a triggered activity, has been reported using radiofrequency energy. Pace-mapping and ventricular activation maps identifying early ventricular activation (20-30 msec before the surface QRS) of spontaneous (idiopathic) and isoproterenolinduced ventricular tachycardia are important techniques used to locate the focus of this tachycardia. Because the right ventricular outflow tract myocardial wall is somewhat thin, care needs to be taken to make sure that lower energies are used to minimize the risk of perforation.
Left ventricular tachycardia. Radiofrequency energy has been used to ablate idiopathic left ventricular tachycardia with right bundle branch block configuration and left axis deviation. This tachycardia is thought to originate from the Purkinje network of the left posterior fascicle. ${ }^{34}$ Pace-mapping with similar QRS is not specific due to capture of the Purkinje fiber network at this site remote from the precise origin of the tachycardia. Nonetheless, radiofrequency energy delivered at this site results in termination of the tachycardia (Figs. 10 and 11).

Ventricular tachycardia following right ventriculotomy. No data are available regarding use of radiofrequency energy to treat ventricular tachycardia following right ventriculotomy; we have used intraoperative excision and cryotherapy, as have others, ${ }^{35}$ to eliminate this arrhythmia. Radiofrequency energy may emerge as an alternative approach.

\section{Immediate Postablation Management and Complications}

Most patients are managed without overnight hospitalization. Chest $X$ ray and echocardiographic followup evaluations are indicated for patients with subclavian or internal jugular vein cannulation, or transseptal approach to the left atrium to rule out pneumothorax or pericardial effusions, respectively, prior to dis- 
$100 \mathrm{~mm} / \mathrm{sec} 100 \mathrm{men} / \mathrm{sec}$

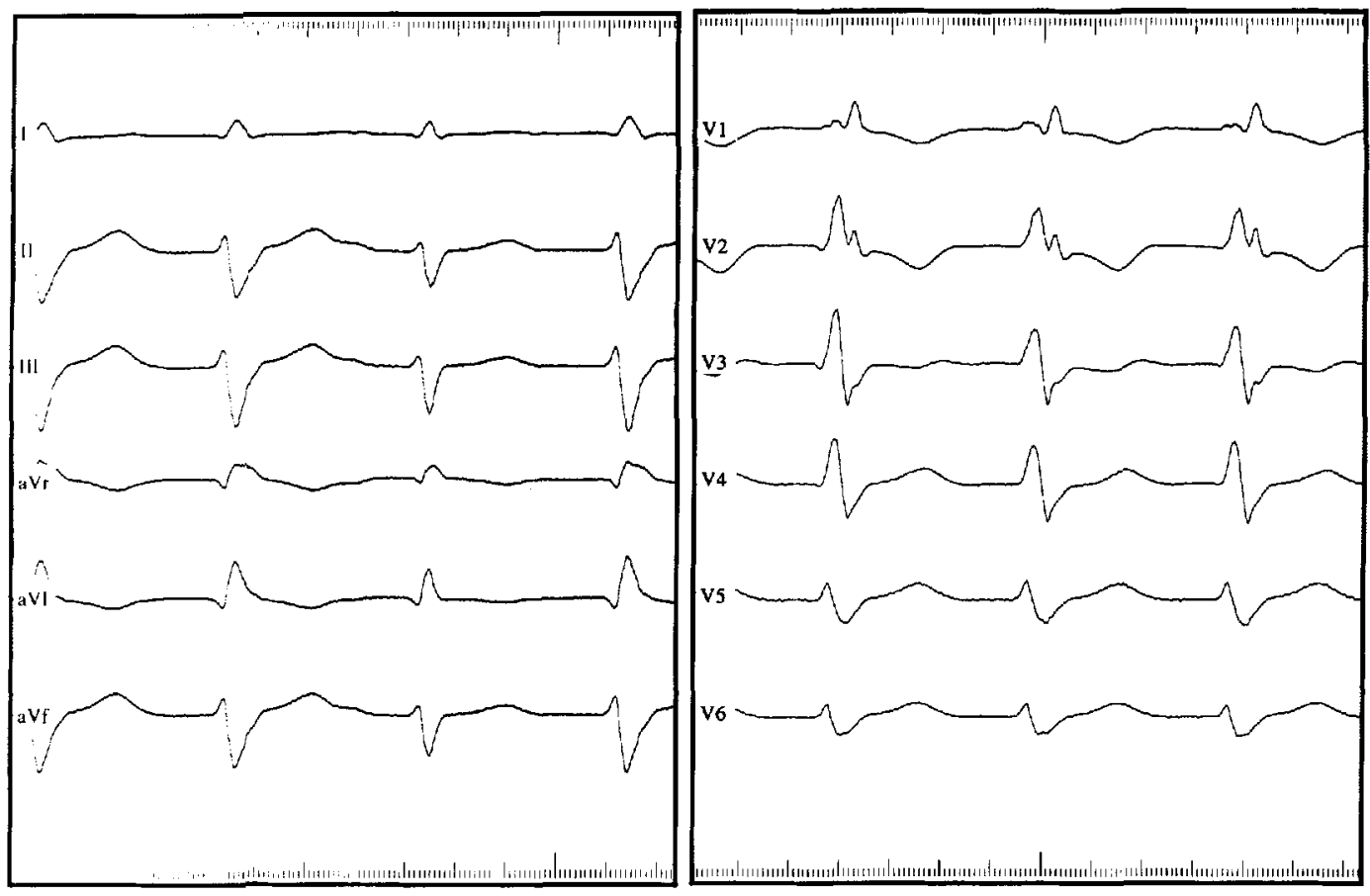

Figure 10. 12-lead ECG of left fascicular ventricular tachycardia. Limb leads I, II, III, aVr, aV1, aVf, and chest leads V1, $\mathrm{V} 2, \mathrm{~V} 3, \mathrm{~V} 4, \mathrm{~V} 5$, and $\mathrm{V} 6$ are shown.

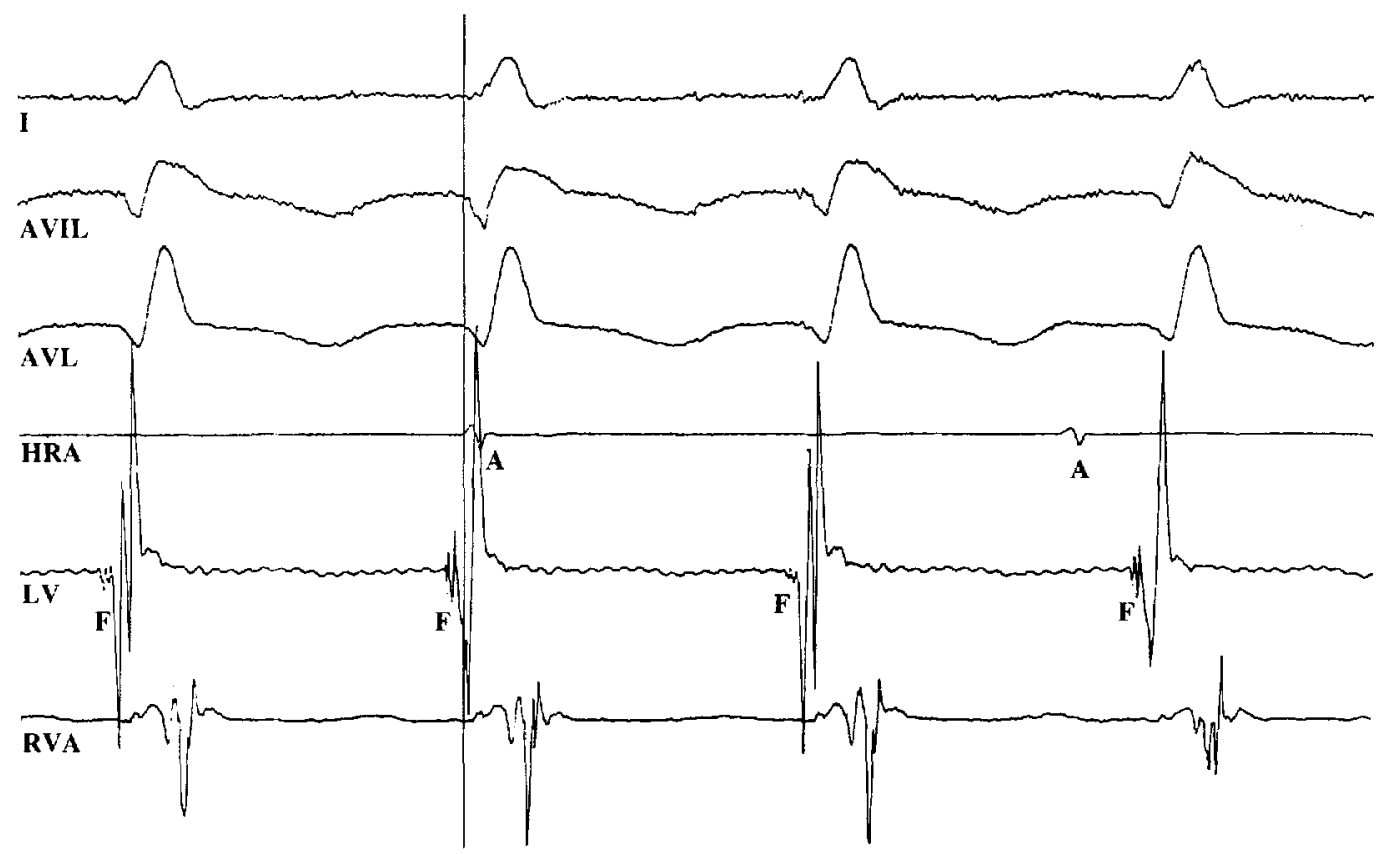

Figure 11. Effective ablation of site of left ventricular tachycardia can be seen. Surface leads I, aVr, aVl, and V5 are shown. Intracardiac electrograms from the right atrium, distal ablation catheter, and His-bundle electrogram can be seen. 
charge that night. Although radiation exposure is of concern and must be minimized, recent data from our institution demonstrate that the radiation dose received during an electrophysiology study and radiofrequency ablation procedure is the same as that received during diagnostic cardiac catherization. ${ }^{36}$ No proarrhythmic effects have been detected up to 5 years following ablation. $^{2}$

A $30 \%$ increase in Doppler detected aortic regurgitation has been reported in young patients for which a retrograde passage of the ablation catheter was used in ablation of left-sided accessory connections. ${ }^{25}$ Experimental data suggested Doppler detected aortic regurgitation is primarily related to the duration of retrograde passage of the catheter across the aortic valve. ${ }^{26}$ Clinical cxperience at the University of Michigan as well as that of others demonstrate absence of aortic regurgitation in paticnts where the transseptal approach was used. ${ }^{27}$

Heart block continues to be a risk for these patients and has been reported to occur in about $2 \%$ of patients undergoing radiofrequency energy application for arrhythmias. ${ }^{3}$ Although lesion size is dependent upon a number of factors, ${ }^{1}$ a recent report has noted increasing ventricular lesion size associated with growth of test lambs, suggesting avoidance of radiofrequency lesions in young myocardium. ${ }^{37}$

\section{Conclusions}

The use of radiofrequency energy for the treatment of arrhythmias in the pediatric population has achieved

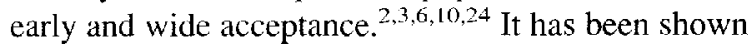
to be safe and effective for a majority of young patients with supraventricular tachycardia. It should be the procedure of choice in patients with manifest Wolff-Parkinson-White syndrome, and also in patients with symptomatic narrow complex tachycardia. It should also be the primary proccdure of choice in patients with automatic atrial tachycardia. Ablation for atrial flutter or sinus node reentry tachycardia in the pediatric age group is promising, but remains unproven in a large group of patients. Success of radiofrequency cnergy for the treatment of atrial fibrillation in children remains unknown; however, atrioventricular node modification or ablation has been shown to be useful in the adult population. ${ }^{17}$ Intractable atrial or junctional arrhythmias have been treated successfully with radiofrequency ablation of the atrioventricular node. Radio- frequency energy has been highly effective for the treatment of idiopathic ventricular tachycardia. Therefore, most children and young adults with symptomatic tachycardia should be strongly considered for radiofrequency ablation of the substrate supporting their tachyarrhythmia.

\section{References}

1. Borggrefe M, Hindricks G, Haverkamp W, et al. Catheter ablation using radiofrequency energy. Clin Cardiol 1990;13: 127-131.

2. Dorastkar PC, Dick M. Current management of supraventricular tachycardia in children. J Heart Dis Stroke 1994;Nov/Dec: $395-400$.

3. Kugler JD, Danford DA, Deal BJ, et al. Radiofrequency catheter ablation for tachyarrhythmias in children and adolescents. N Engl J Med 1994;330:1481-1487.

4. Calkins H, Langberg J, Sousa J, et al. Radiofrequency catheter ablation of accessory atrioventricular connections in $250 \mathrm{pa}-$ tients. Abbreviated therapeutic approach to Wolff-ParkinsonWhite syndrome. Circulation 1992;85:1337-1346.

5. Dick M, O'Connor B, Serwer GA, et al. Use of radiofrequency current to ablate accessory connections in children. Circulation 1991;84:2318-2324.

6. Van Hare GF, Lesh MD, Scheinman M, et al. Percutaneous radiofrequency catheter ablation for supraventricular arrhyth mias in children. JACC 1991;17:1613-1620.

7. Langberg JJ, Leon A, Borganelli $\mathbf{M}$, et al. A randomized, prospective comparison of anterior and posterior approaches to radiofrequency catheter ablation of atrioventricular nodal reentry tachycardia. Circulation 1993;87:1551-1556.

8. Lee MA, Morady F, Kadish A, et al. Catheter modification of the atrioventricular junction with radiofrequency energy for control of atrioventricular nodal reentry tachycardia. Circulation 1991;83:827-835.

9. Jackman WM, Beckman KJ, McClelland JH, et al. Treatment of supraventricular tachycardia due to atrioventricular nodal reentry by radiofrequency cathcter ablation of slow-pathway conduction. N Engl J Med 1992;327:313-318.

10. Walsh EP, Saul P, Hulse E, et al. Transcatheter ablation of ectopic atrial tachycardia in young patients using radiofrequency current. Circulation 1992;86:1138-1146.

11. Stevenson WG, Nademanee K, Weiss JN, et al. Treatment of catecholamine-sensitive right ventricular tachycardia by endocardial catheter ablation. JACC 1990;16:752-755.

12. Nakagawa $\mathbf{H}$, Beckman KJ, McClelland JH, et al. Radiofrequency catheter ablation of idiopathic left ventricular tachycardia guided by a purkinje potential. Circulation 1993;88: $2607-2617$

13. Lesh MD, Van Hare GF, Fitzpatrick AP, et al. Curing reentrant atrial arrhythmias. Targeting protected zones of slow conduction by catheter ablation. J Electrocardiol 1993;26(Suppl): 194-203.

14. Lesh MD, Van Hare GF, Epstein LM, et al. Radiofrequency catheter ablation of atrial arrhythmias. Results and mechanisms. Circulation 1994;89:1074-1089.

15. Urcelay G, Dick M II, Bove EL, et al. Intraoperative mapping and radiofrequency ablation of the His bundlc in a patient with complex congenital heart disease and intractable atrial arrhythmias following the Fontan operation. PACE 1993;16: $1437-1440$ 


\section{DOROSTKAR AND DICK}

16. Russell MW, Dorostkar PC, Dick M II, et al. Percutaneous catheter interruption of atrioventricular conduction using radiofrequency energy in a paticnt with transposition of the great arteries and intractable atrial arrhythmias following the Mustard operation. PACE (In press)

17. Williamson BD, Man KC, Daoud E, et al. Radiofrequency catheter modification of atrioventricular conduction to control the ventricular rate during atrial fibrillation. $N$ Engl J Med 1994:331(14):910-917.

18. O'Connor BK, Dick M. What every pediatrician should know about supraventricular tachycardia. Pediatric Annals 1991;20: $368-376$

19. Calkins H, Kim YN, Schmaltz S, ct al. Electrogram criteria for identification of appropriate target sites for radiofrequency catheter ablation of accessory atrioventricular connections. Circulation $1992 ; 85: 565-573$.

20. Schwartz SM, Dick M, Dorostkar PC, et al. Electrogram patterns associated with successful radiofrequency ablation of accessory pathways in children. Pediatric Cardiology (In press).

21. Borganelli M, El-Atassi R, Leon A, et al. Determinants of impedance during radiofrequency catheter ablation in humans. Am J Cardiol 1992;69:1095-1097.

22. Fitzpatrick AP, Lesh MD. A new statistical appraisal of baseline ECG features for localization of accessory pathways. J Electrocardiol $1993 ; 26$ (Suppl):220-226.

23. Fitzpatrick AP, Gonzales RP, Lesh MD, et al. New algorithm for the localization of accessory atrioventricular connections using a baseline ECG. JACC 1994;23(5):1272.

24. Saul JP, Hulse JE, Wang DE, et al. Catheter ablation of accessory atrioventricular pathways in young patients: Use of long vascular sheaths, the transseptal approach and a retrograde left posterior parallel approach. JACC 1993;21:571-583.

25. Minich LL, Snider AR, Dick M. Doppler detection of valvular regurgitation after radiofrequency ablation of accessory connections. Am J Cardiol 1992;70:116-117.

26. Dorostkar PC, Tacy TA, Urcelay G, et al. Doppler detected aortic regurgitation following retrograde passage of an electrode catheter into the left ventricle is primarily a function of duration of catheter placement across the aortic valve. (abstract) PACE 1994;17:847.
27. Dorostkar PC, Lloyd TR, Vermilion R, et al. Prevention of aortic and mitral regurgitation during radiofrequency ablation of left-sided accessory connections in children using the transseptal approach. (abstract) PACE 1994; 17:848.

28. Dorostkar PC, Silka MJ, Sewer GA, et al. Radiofrequency ablation for the treatment of persistent junctional reciprocating tachycardia in children. (abstract) Am J Cardiol 1992;70(4): 561.

29. Epstcin ML, Benditt DG. Long-term evaluation of persistent supraventricular tachycardia in children: Clinical and electrocardiographic features. Am Heart J 1981;102:80-84.

30. Chien WW, Cohen TJ, Lee MA, et al. Electrophysiological findings and long-term follow-up of patients with the permanent form of junctional reciprocating tachycardia treated by catheter ablation. Circulation 1992;85(4):1329-1336.

31. Kuck KH, Schluter M. Single-catheter approach to radiofrequency current ablation of left-sided accessory pathways in patients with Wolff-Parkinson-White syndrome. Circulation $1991 ; 84: 2366-2375$.

32. Bromberg BI, Dick M, Snider AR, et al. Tachycardia related cardiomyopathy in children: Response to control of the arrhythmia. J Interven Cardiol 1989;2:21 1-218.

33. Chu E, Fitzpatrick AP, Chin MC, et al. Radiofrequency cathcter ablation guided by intracardiac echocardiography. Circulation 1994:89(3):1301-1305.

34. Nakagawa $\mathbf{H}$, Beckman KJ, McClelland $\mathbf{J H}$, et al. Radiofrequency catheter ablation of idiopathic left ventricular tachycardia guided by a Purkinje potential. Circulation 1993;88: 2607-2617.

35. Misaki T, Tsubota M, Watanabe G, et al. Surgical treatment of ventricular tachycardia after surgical repair of tetralogy of Fallot. Relation between intraoperative mapping and histological findings. Circulation 1994;90:264-271.

36. Kowalski CA, Dorostkar PC, Miklos JA, et al. Comparison of radiation dose received during electrophysiology study and radiofrequency ablation or cardiac catheterization in children. (abstract) Circulation 1994;90:I-99.

37. Saul JP, Hulse JE, Papagiannis J, et al. Late enlargement of radiofrequency lesions in infant lambs. Implications for ablation procedures in small children. Circulation 1994;90: $492-499$. 